\title{
Mesoscale modeling of mechanics of carbon nanotubes: Self-assembly, self-folding, and fracture
}

\author{
Markus J. Buehler ${ }^{a}$ \\ Department of Civil and Environmental Engineering, Massachusetts Institute of Technology, \\ Cambridge, Massachusetts, 02139
}

(Received 30 March 2006; accepted 23 June 2006)

\begin{abstract}
Using concepts of hierarchical multiscale modeling, we report development of a mesoscopic model for single-wall carbon nanotubes with parameters completely derived from full atomistic simulations. The parameters in the mesoscopic model are fit to reproduce elastic, fracture, and adhesion properties of carbon nanotubes, in this article demonstrated for $(5,5)$ carbon nanotubes. The mesoscale model enables modeling of the dynamics of systems with hundreds of ultralong carbon nanotubes over time scales approaching microseconds. We apply our mesoscopic model to study self-assembly processes, including self-folding, bundle formation, as well as the response of bundles of carbon nanotubes to severe mechanical stimulation under compression, bending, and tension. Our results with mesoscale modeling corroborate earlier results, suggesting a novel self-folding mechanism, leading to creation of racket-shaped carbon nanotube structures, provided that the aspect ratio of the carbon nanotube is sufficiently large. We find that the persistence length of the $(5,5)$ carbon nanotube is on the order of a few micrometers in the temperature regime from 300 to $1000 \mathrm{~K}$.
\end{abstract}

\section{INTRODUCTION}

Carbon nanotubes (CNTs) constitute a prominent example of nanomaterials, with many potential applications that could take advantage of their unique mechanical, electrical, and optical properties. ${ }^{1-6}$ A fundamental understanding of the properties of individual CNTs, assemblies of CNTs in bundles or nanopillars, ${ }^{7}$ or CNTs in conjunction with biological molecules such as $\mathrm{DNA}^{8}$ may enable new technologies and to engineer CNTbased devices.

In particular, the mechanical properties of CNTs could be important in many applications, including cases in which the primary role of CNTs is not related to their mechanical properties. Nevertheless, a thorough understanding of the mechanical properties is essential in designing manufacturing processes or to ensure reliability during operation of devices.

The interactions of individual CNTs can play a critical role in application and during fabrication processes and may pose significant challenges compared with macroscopic classical engineering applications. This is because at the nanoscale, weak dispersive van der Waals interactions ( $\mathrm{vdW}$ ) play a more prominent role and often govern the mechanics or self-assembly dynamics of those

\footnotetext{
a) Address all correspondence to this author.

e-mail: mbuehler@MIT.EDU

DOI: 10.1557/JMR.2006.0347
}

materials. The interplay of such adhesive forces with covalent bonding within CNTs is not well understood for many CNT systems.

\section{A. Review: Modeling of mechanical behavior of CNTs}

The mechanics of carbon nanotubes has been discussed in various articles published over the last decade, from both a continuum and an atomistic perspective..$^{9-13}$ Most studies in the literature that focused on CNTs have considered only small-aspect ratio CNTs or a small number of interacting CNTs. In contrast, experiments have shown that single wall carbon nanotubes (SWNTs) can grow to lengths above $700 \mathrm{~nm}$ with a diameter of $0.9 \mathrm{~nm}$, resulting in an aspect ratio as large as about $800 .{ }^{2} \mathrm{CNTs}$ with ultrasmall diameters approaching $0.4 \mathrm{~nm}$ have been shown to be stable, both in experiments and by computation. ${ }^{14,15}$

In a classical article by Yacobsen and co-workers, the behavior of single, freestanding single-wall carbon nanotubes (SWNTs) under compressive loading was investigated using classical, molecular-dynamics (MD) with empirical potentials. ${ }^{16}$ The longest tube considered was 6 $\mathrm{nm}$ with a diameter of $1 \mathrm{~nm}$. The authors developed a continuum shell model to describe the buckling modes of the CNTs.

Ozaki and co-workers ${ }^{17}$ investigated SWNTs under axial compression using tight binding (TB) MD methods 
and system sizes up to a few thousand atoms. In their studies, the length of the nanotubes was limited to about $14 \mathrm{~nm}$ with a diameter of $0.67 \mathrm{~nm}$. A ripple shell buckling was observed once the SWNT was put in compressive loading. The details of the ripple buckling (e.g., the ripple wavelength) were found to be strongly dependent on the temperature, and the stress under large strain and zero temperature depends on the helicity. SWNTs under tensile and compressive loading were studied by Dereli and Ozdogan ${ }^{18}$ using a TB-MD scheme in an attempt to obtain the stress-strain curve, theoretical strength, and Poisson's ratio for SWNTs. The authors modeled CNTs with about 400 atoms, featuring a total length of 20 layers, corresponding to a few nanometers.

$\mathrm{Ru}^{19}$ considered buckling of a double-walled carbon nanotube embedded in an elastic medium under compressive loading using a double-shell continuum mechanics model. The main finding was that critical buckling strain for MWNTs may be reduced compared to SWNTs, indicating that MWNT could even be more susceptible to axial buckling than SWNTs. Other research focused on the mechanical properties of CNTs filled with small molecules. The authors in Ref. 20 investigated compression of CNTs filled with nano-particles and molecules (e.g., $\mathrm{C}_{60}, \mathrm{NH}_{4}$ ). The longest tube considered had a length around $20 \mathrm{~nm}$.

Research has also been carried out to investigate the elastic properties of CNTs. As recently shown by Hod and coworkers, ${ }^{21}$ SWNTs can be bent into closed-ring structures ("nano-rings"). The Tersoff-Brenner potential was used within a classical MD scheme to study the elastic properties of such CNT ring structures. There are also investigations focused on the interaction between different CNTs and their adhesive properties. It was shown that when SWNTs are formed into bundles due to van der Waals (vdW) interaction, ${ }^{22}$ their cross-section shape can change significantly. The change in shape can modify the flexural rigidity and promote bending or other forms of elastic deformation. Interactions of CNTs with surfaces can also change their shape. ${ }^{23}$ The shape change can also affect the electrical conductivity of CNTs, clearly indicating the immediate link of CNT mechanics and other nonmechanics applications. Failure mechanisms such as fracture nucleation in SWNTs under tension have been discussed using combined continuumatomistic approaches. ${ }^{9,24-26}$

Several other studies using molecular dynamics have been carried out to develop a molecular level understanding of the failure processes, using a variety of atomistic modeling techniques. ${ }^{9,10,27-31}$

\section{B. Mechanics of assemblies of CNTs}

A rigorous understanding of the mechanical behavior of CNTs originating from the atomistic or molecular origin and the properties of assemblies of a large number of CNTs has not been established up to date. However, this scale is critical in enabling technological applications and usage of CNTs as basis for new materials.

Full atomistic models have proven to be a quite useful approach in understanding the mechanical behavior of CNTs. ${ }^{9,10,27-31}$ However, such models are limited to very short time- and length-scales so that a direct comparison with experimental scales is often extremely difficult or impossible.

To overcome those limitations, we propose developing a mesoscale model of CNTs by reducing some of the atomistic degrees of freedom, representing CNTs as a collection of beads connected by spring-like molecular multi-body interatomic potentials. These interaction potentials describe the resistance to tensile load, bending, and interaction between different CNTs. Our mesoscale model is capable of treating the deformation physics of large assemblies of carbon nanotubes corresponding to systems with millions of atoms, while incorporating nonlinear elasticity, fracture behavior, and adhesion properties between different CNTs, ranging through time scales of several microseconds. With parameters rigorously derived from full atomistic simulations, our mesoscale model provides a first-principles-based description of CNTs. Because of the accessible time and length scales, such models may significantly contribute to development of a fundamental understanding of cross-scale interactions.

Bead-type models have been implemented for several other molecular systems and applications..$^{32-35}$ Mesoscale models, with information about chemistry of bond rupture, enable modeling of the complex interaction of chemical bonds of different strengths. Mesoscale models may also enable studies of CNTs interacting with matrix materials, which is so far mainly addressed using experimental techniques. ${ }^{36}$

\section{Motivation and outline}

The focal points of this paper are self-assembly processes of CNTs, as well as properties and response of self-assembled structures under mechanical stimulation. ${ }^{37}$ Self-assembly processes of CNTs play critical roles in manufacturing nanodevices, as has been demonstrated in several experimental studies.

For example, growth of nano-pillars of CNTs has recently been reported, involving MWCNTs of lengths up to $500 \mathrm{~nm}$ and more. ${ }^{7}$ Similar mechanisms have been reported in other works discussing self-assembly processes of MWCNTs creating structures with lengths of several micrometers to meters. ${ }^{38}$ In several recent studies, self-assembly of nanostructures has been discussed in light of combination with biological mechanisms and concepts. ${ }^{39}$ Several other self-assembly processes 
involving CNTs have been reported. ${ }^{40,41}$ Our modeling is further motivated by recent MD results that suggest the existence of self-folded racket-like CNT structures, ${ }^{42,43}$ as shown in Fig. 1. So far, only full atomistic studies have been carried out and have been somewhat limited by the accessible time scale, in particular in describing the slow motions of the ultralong CNTs.

Computer models that allow straightforward access to the properties and mechanisms of large-scale assembles of CNTs could provide immediate help in engineering those structures and materials.

The outline of this paper is as follows. In Sec. II, we discuss a series of full atomistic calculations to determine fundamental mechanical parameters of a $(5,5)$ singlewall CNT, including tensile stiffness, bending stiffness, persistence length, and adhesion properties. In Sec. III, we discuss a simple mesoscopic model and derivation of parameters for this model from full atomistic studies. Section IV is dedicated to application of the mesoscale model to demonstrate its usefulness. Examples include studies of self-folding mechanics, self-assembly of two CNTs into conglomerated structures, and self-assembly and properties of bundles of CNTs. The results and implications of this model are discussed in Sec. V.

\section{ELASTIC AND FRACTURE PROPERTIES: ATOMISTIC MODELING}

In this section, we describe a series of mechanical loading cases to determine parameters for our mesoscopic model. These studies consist of the following three loading cases: (i) tensile loading, to determine Young's modulus, change of Young's modulus as a
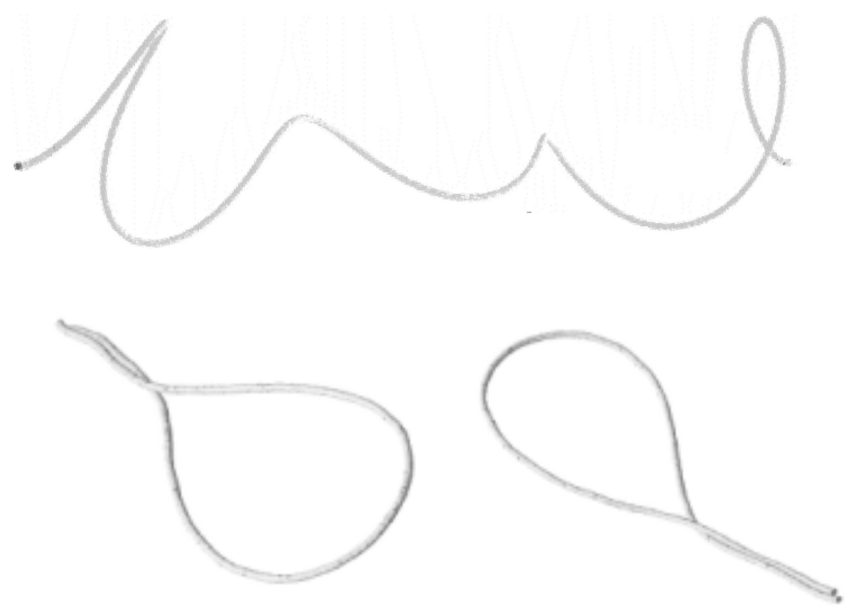

FIG. 1. Full atomistic calculations of the properties of ultralong CNTs as reported in Refs. 42 and 43. Above a critical aspect ratio, CNTs self-fold into racket-like structures. It can be observed that the selfaligned part connects to the free length of the CNT in a characteristic way by crossing over at an angle of around $90^{\circ}$. We believe that this phenomenon could be explained due to the reduced energy by increasing the contact length in this characteristic manner. function of deformation, and fracture stress and strain; (ii) bending to determine the bending stiffness of CNTs; and (iii) an assembly of two CNTs to determine their adhesion energy. The different loading cases are summarized in Figs. 2(a) and 2(b).

All studies were carried out using $(5,5)$ armchair single wall CNTs (SWCNTs). This particular CNT is chosen based on earlier full atomistic studies of tubes with this geometry $^{42,43}$ (see Fig. 1).

\section{A. Classical molecular dynamics simulation method}

The atomistic studies were carried out using classical MD. ${ }^{44}$ MD predicts the motion of a large number of atoms by numerically integrating the equations of motion governed by interatomic interaction. Normally, it is necessary to rely on classical MD to simulate system sizes above a few thousand atoms and time-scales on the order of nanoseconds, as such system sizes and time scales are still far beyond the capabilities of quantum mechanics based methods.

Interatomic potentials are the core of classical MD methods. During recent decades, numerous potentials describing atomic interaction in various materials with different levels of accuracy have been proposed, each having unique problems and strengths. For covalently bonded materials like carbon or silicon, bond-order multi-body potentials have been developed (e.g., Tersoff

(a)

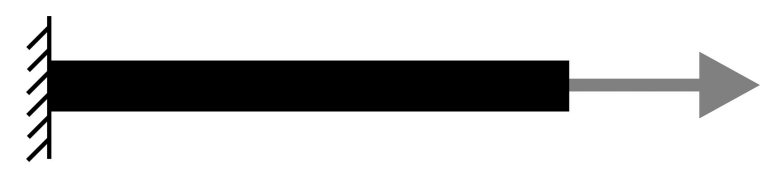

(b)

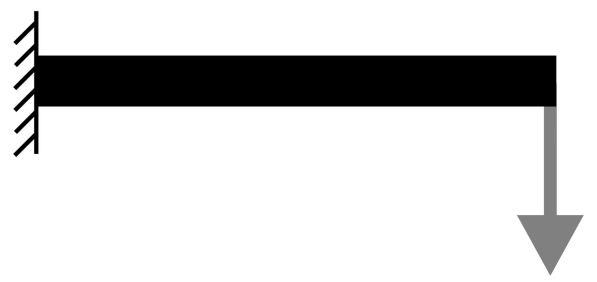

(c)

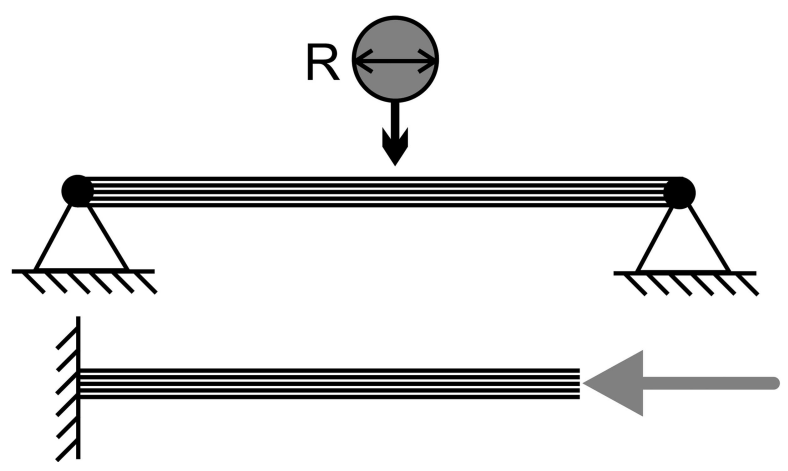

FIG. 2. (a, b) Mechanical lading cases of the CNT to derive parameters for the mesoscopic model. (c) Setup for the indentation bending test carried out with the mesoscopic model. (d) Setup for compression experiments of bundles of CNTs. 
potential or Stillinger-Weber potential ${ }^{45,46}$ ). These multi-body potentials capture not only pair-wise interactions but also additional contributions from the local geometric configuration of the neighboring atoms. Here we use the Tersoff potential ${ }^{45}$ to describe the interatomic bonding of $\mathrm{C}-\mathrm{C}$ atoms. The Tersoff potential has proven to be a reliable empirical potential to describe the bonding inside carbon nanotubes, in particular around the equilibrium position. For the vdW interaction between non-bonding atoms, we use Lennard-Jones 12:6 potential $^{15}$ with parameters $\epsilon=3.851 \AA$ and $\sigma=0.0040 \mathrm{eV}$.

The time step was chosen to be on the order of $10^{-15}$ seconds. Whereas the vdW interaction features a larger cutoff of $15 \AA$, the Tersoff potential has a cutoff of $2.1 \AA$. The MD calculations were preformed using the IMD (Institute for Theoretical and Applied Physics Molecular Dynamics Code) code. ${ }^{47,48}$

\section{B. Tensile loading}

\section{Elastic properties}

The computational experiments to model tensile deformation of a CNT is implemented by keeping one end of the CNT fixed, while slowly displacing the other end in the axial direction of the tube. The loading case is shown in Fig. 2(a).

As the tube was stretched, we calculated the virial stress $^{49}$ averaged over the complete tube volume. We assumed a circular cross-sectional area $A_{\mathrm{C}}=\pi \mathrm{r}^{2}$, assuming that $R \approx 3.5 \AA$. The stress tensor component in the loading direction was used to extract information about the stress as a function of applied uniaxial strain. The stress-strain curve was then used to determine Young's modulus $E$, defined as

$$
E(\epsilon)=\frac{\partial \sigma}{\partial \epsilon} \approx \frac{\Delta \sigma}{\Delta \epsilon}
$$

Note that Young's modulus is typically dependent on the strain $\epsilon$, showing a decrease with increasing strain due to softening of chemical bonds as their breaking point is reached. This phenomenon is referred to as hyperelasticity. Young's modulus is independent of the length of the molecule.

For small deformation, we estimated Young's modulus of the $(5,5) \mathrm{CNT}$ to be around $E \approx 2 \mathrm{TPa}$. Figure 3 depicts the stress-strain plot (a) and an analysis of the Young's modulus as a function of strain (b). The stress-strain plot shown in Fig. 3(a) contains results of calculations carried out with three different displacement loading rates, 1.6, 0.4 , and $0.2 \mathrm{~km} / \mathrm{s}$ (corresponding to strain rates of $1.25 \times$ $10^{11}, 3.2 \times 10^{10}$, and $1.6 \times 10^{10} \mathrm{~s}^{-1}$ ). Whereas the result differs significantly for the largest loading rate, the results for the slower rates are similar, indicating convergence of the fracture properties. Figure 3(b) depicts the result only for the loading rate $0.2 \mathrm{~km} / \mathrm{s}$.
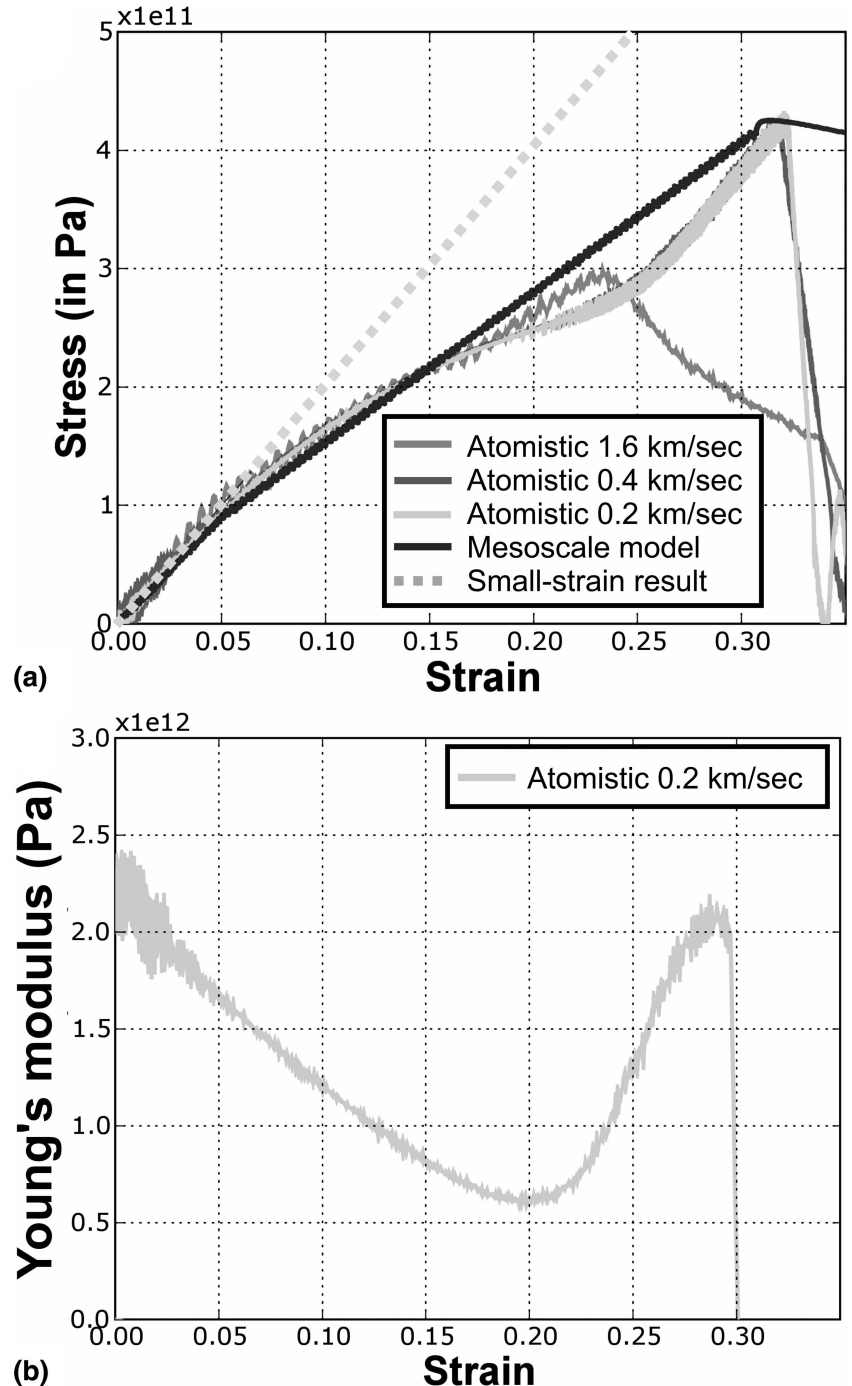

FIG. 3. (a) Stress versus strain and (b) local Young's modulus for stretching a $(5,5)$ CNT using the Tersoff potential. Young's modulus decreases with increasing strain but shows a small and shallow peak close to the breaking point. The dotted line is the tangent stress-strain law for small deformation. The stress-strain plot contains results with three different loading rates, $1.6,0.4$, and $0.2 \mathrm{~km} / \mathrm{s}$. While the result differs significantly for the largest loading rate, the results for the slower rates are similar. The dark line is the result using the mesoscopic model. (b) Result for the loading rate $0.2 \mathrm{~km} / \mathrm{s}$.

\section{Fracture properties}

Deformation of the CNTs is elastic only for small deformation. When extremely large strains were applied to the carbon nanotube, we observed formation of defects that eventually lead to fracture of the carbon nanotube. The fracture strain of the CNT was $\epsilon_{\mathrm{F}} \approx 32 \%$, as can be verified in Fig. 3. Fracture of the CNT is accompanied by a zero slope in the stress-strain plot, a behavior also found in atomic crystals.

Fracture of the CNT initiates by generation of localized shear defects in the hexagonal lattice of the CNT, somewhat reminiscent of Stone-Wales defects. ${ }^{28,50,51}$ 
These mechanisms occur within a fraction of a percent strain away from the peak in tensile stress in the stressstrain plot [Fig. 3(a)]. These localized defects quickly lead to formation of microcracks that lead to macroscopic failure of the carbon nanotube. The hexagonal lattice remains intact up to strains very close to macroscopic failure. Several snapshots of the atomistic fracture mechanics are shown in Fig. 4.

The change in slope in the stress-strain plot [Fig. 3(a), deviation from linear elastic regime starting at around $5 \%$ tensile strain] is due to homogeneous deformation of the entire lattice in the CNT, not to development of local defects.

\section{Bending modulus and persistence length}

\section{Cantilever bending test}

Similar to the tensile test, we performed a simple computational experiment to describe the bending of a CNT by clamping it at the outermost left boundary and

(a)

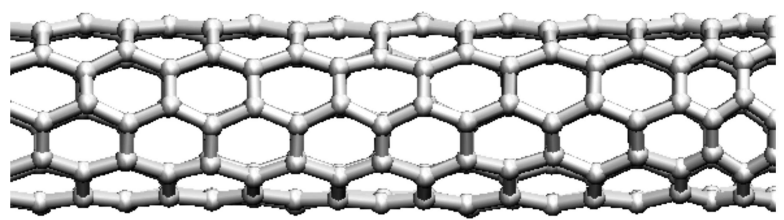

(b)

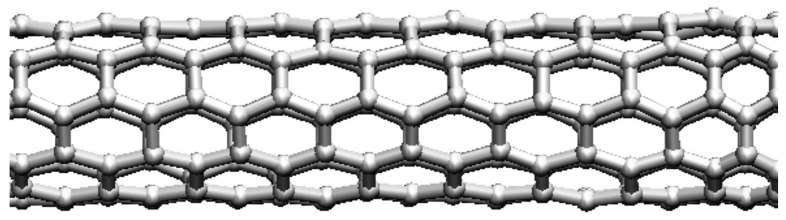

(c)

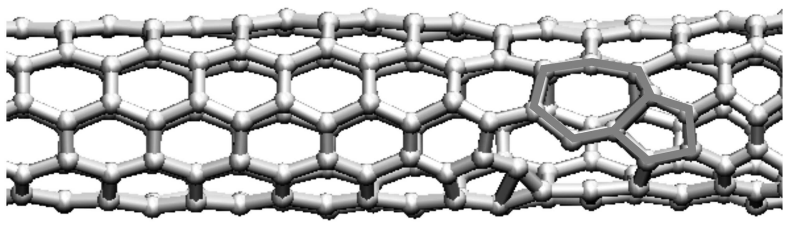

(d)

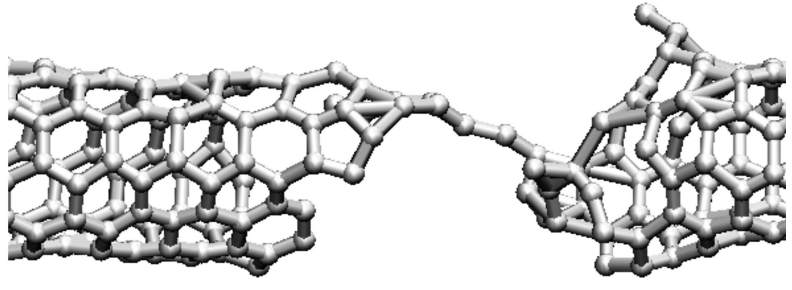

FIG. 4. Fracture mechanics of a $(5,5) \mathrm{CNT}$, modeled using the Tersoff potential. (a)-(d) Atomistic mechanics as the lateral tensile strain is increased. Fracture initiates by generation of localized shear defects in the hexagonal lattice of the CNT, somewhat reminiscent of 5-7 StoneWales defects, ${ }^{28,50,51}$ indicated in (c) by the lines, showing the 7-membered ring next to the 5-membered ring. These localized defects quickly lead to formation of microcracks that lead to macroscopic disintegration of the carbon nanotube. (d) Formation of a linear Catom chain at the final stages of fracture. Failure initiates close to the boundaries of the CNT, possibly due to the clamped boundary conditions. applying a force at the right end of the CNT, as shown in Fig. 2(b).

Atoms within a region of $5 \AA$ in the outermost, left part of the CNT are held fixed in all directions, resembling the clamped boundary condition used in continuum theory. By measuring the bending displacement $d$, the bending stiffness $E I$ is then given by

$$
E I=\frac{F_{\mathrm{app}} L^{3}}{3 d}
$$

From the clamped bending test, we find that $E I=$ $6.65 \times 10^{-26} \mathrm{Nm}^{2}$ for the $(5,5) \mathrm{CNT}$.

\section{Persistence length}

Because we are interested in the molecular dynamics of ultralong CNTs at elevated temperature, entropic effects may begin to play an important role. The persistence length describes the molecular length at which the thermal energy becomes sufficient to induce significant bending in the CNT, even without application of external forces.

The persistence length is defined as

$$
\xi=\frac{E I}{\mathrm{k}_{\mathrm{B}} T}
$$

where $\mathrm{k}_{\mathrm{B}}$ is the Boltzmann constant, and $T$ is the temperature. At $T=300 \mathrm{~K}$, we find the persistence length $\zeta \approx 1.61 \times 10^{-5} \mathrm{~m}$, reducing to a few micrometers at temperature around $1000 \mathrm{~K}$.

This result suggests that most CNTs produced by experimental techniques are below the persistence length. CNTs with larger diameter or multiwalled CNTs typically have an even larger bending stiffness, leading to larger persistence length.

We note that the CNT is a few orders of magnitude stiffer than many biological molecules (for example, the bending stiffness of a tropocollagen molecule is $E I=$ $\left.9.71 \times 10^{-29} \mathrm{Nm}^{2}\right) .{ }^{52}$

\section{Interaction of multiple CNTs: Adhesion properties}

Because we are interested in the self-assembly of CNTs, we investigated the interaction of multiple CNTs. Primary interaction forces are weak dispersive interactions, such as van der Waals forces. We assume that no covalent bonds may form between different CNTs.

\section{Equilibrium distance between two CNTs}

The equilibrium distance of the CNTs is denoted by $\Delta D$. The parameter $\Delta D$ is determined from full atomistic simulations and is found to be $\Delta D \approx 10.5 \AA$. We have also confirmed that assemblies of several CNTs form 
triangular lattices. Our molecular dynamics simulation results suggest that pair-wise interaction between different CNTs represents a reasonable model.

\section{Adhesion energy}

The adhesion energy of two $(5,5)$ tubes is determined to be $E_{\mathrm{L}}=2.31 \times 10^{-10} \mathrm{~J} / \mathrm{m}$.

\section{MESOSCALE MODEL DEVELOPMENT}

Here we discuss our method in deriving parameters for a mesoscale bead-type model of CNTs from full atomistic calculations (see the schematic illustration in Fig. 5). This derivation is based completely on the results reported in Sec. II, without additional empirical or fitting parameters.

\section{A. Model development: Training from pure atomistic results using energy and force matching}

Our goal is to develop a simple mesoscopic model to perform large-scale studies of the mechanics of CNTs. We express the total energy of the system as

$$
E=E_{\mathrm{T}}+E_{\mathrm{B}}+E_{\text {weak }}
$$

where $E_{\mathrm{T}}$ is the energy stored in chemical bonds due to stretching along the axial direction, $E_{\mathrm{B}}$ is the energy due to bending of the CNT, and $E_{\text {weak }}$ constitutes weak (vdW) interactions. The total energy contribution of each part is given by the sum over all pair-wise and triple (angular) interactions in the system; thus

$$
E_{\mathrm{T}, \text { weak }}=\sum_{\text {pairs }} \phi_{1}(r)
$$

for the tensile and weak interactions (both summed pairwise), and

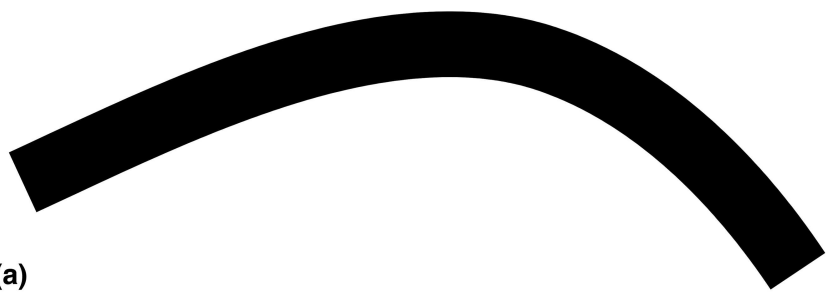

(a)

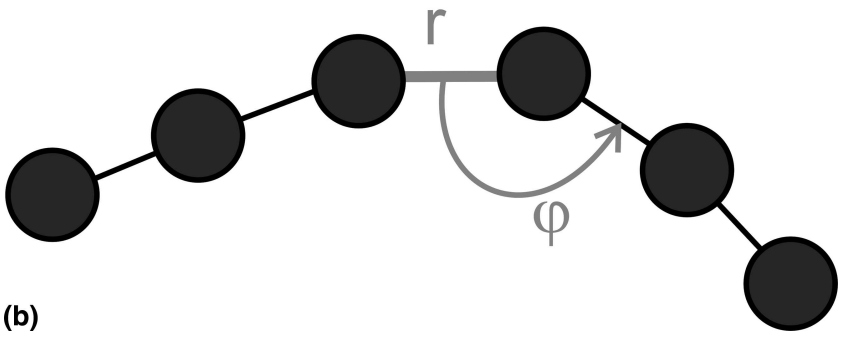

FIG. 5. Atomistic and corresponding mesoscopic model of a CNT (schematic). (a) The atomistic representation of the CNT is replaced by (b) a collection of beads interacting with various molecular potentials.

$$
E_{\mathrm{B}}=\sum_{\text {angles }} \phi_{\mathrm{B}}(\varphi)
$$

summed over all triples of particles in the systems. The bending energy is given by

$$
\phi_{\mathrm{B}}(\varphi)=\frac{1}{2} \mathrm{k}_{\mathrm{B}}\left(\varphi-\varphi_{0}\right)^{2},
$$

with $\mathrm{k}_{\mathrm{B}}$ as the spring constant relating to the bending stiffness and $\varphi$ as the bending angle between three particles. Calculation of the angle thus requires consideration of the position of three atoms. The molecular potential is thus a three-body potential.

We approximate the nonlinear stress-strain behavior under tensile loading with a bilinear model that has been used successfully in earlier studies of fracture ${ }^{53,54}$ [the function $E_{\mathrm{T}}$ is given by integrating $F_{\mathrm{T}}(r)$ along the radial distance]. The force between two particles is $F_{\mathrm{T}}(r)=$ $-\partial \phi_{\mathrm{T}}(r) / \partial r$, where

$$
-\frac{\mathrm{d} \phi_{\mathrm{T}}}{\mathrm{d} r}(r)=H\left(r_{\text {break }}-r\right)\left\{\begin{array}{ll}
k_{\mathrm{T}}^{(0)}\left(r-r_{0}\right) & \text { if } r<r_{1} \\
k_{\mathrm{T}}^{(1)}\left(r-\tilde{r}_{1}\right) & \text { if } r \geqslant r_{1}
\end{array} .\right.
$$

In Eq. (8), $H\left(r-r_{\text {break }}\right)$ is the Heaviside function $H(a)$, which is defined to be zero for $a<0$, and one for $a \geqslant 0$, and $k_{\mathrm{T}}^{(0)}$ as well as $k_{\mathrm{T}}^{(1)}$ for the small and large-deformation spring constants. The parameter $\tilde{r}_{1}=r_{1}-k_{\mathrm{T}}^{(0)} / k_{\mathrm{T}}^{(1)}\left(r_{1}-\right.$ $\left.r_{0}\right)$ is obtained from force continuity conditions.

This model is chosen to reproduce the nonlinear elastic and fracture behavior of carbon nanotubes. The availability of two spring constants enables modeling changes in the elastic properties due to increasing deformation. The Heaviside function allows description of the drop of forces to zero at the initiation of fracture of the carbon nanotube.

In addition, we assume weak, dispersive interactions between either different parts of each molecule or different molecules, defined by a Lennard-Jones 12:6 (LJ) function

$$
\phi_{\text {weak }}(r)=4 \epsilon\left(\left[\frac{\sigma}{r}\right]^{12}-\left[\frac{\sigma}{r}\right]^{6}\right),
$$

with $\sigma$ as the distance parameter and $\epsilon$ describing the energy well depth at equilibrium. The LJ potential has been shown to be a good model for such dispersive interactions (see, for example, Ref. 55).

\section{Equilibrium distances of beads and corresponding masses}

The mass of each bead is determined by assuming a homogeneous distribution of mass in the molecular model. Given the homogeneous structure of CNTs, this is a reasonable approximation. We assume an equilibrium 
distance of two particles of $r_{0}=10 \AA$. The mass per unit length in a $(5,5)$ CNT is given by

$$
\rho_{(5,5)}=195.323 \mathrm{amu} / \AA \text {. }
$$

Based on this mass density and the equilibrium distance between particle, the mass of a single particle is given by $M_{(5,5)}^{r_{0}=10 \AA}=1953.23 \mathrm{amu}$.

\section{Dispersive and nonbonding interactions}

The LJ parameters are chosen to reproduce the adhesion energy determined from full atomistic simulations. In all these considerations, we assume that a pair-wise interaction between different particles is sufficient and that there are no multi-body contributions.

Based on these assumptions, we model the interactions between different molecules using a LJ 12:6 potential.

The equilibrium bond distance is related to the distance $\Delta D$ between two CNTs in contact by vdW interactions. With

$$
\phi=\tan ^{-1}\left(\frac{r_{0}}{2 \Delta D}\right)
$$

we arrive at a relationship between $\Delta D$ and the angle $\phi$ that depends on the geometry of the mesoscopic system,

$$
D=\frac{\Delta D}{\cos \phi} .
$$

The distance parameter $\sigma$ is then given by

$$
\sigma=\frac{D}{\sqrt[6]{2}} \approx 11.63 \AA \text {, }
$$

where $D$ is the equilibrium bond distance.

The potential minimum is at $r=D$ and is given by $-\epsilon$. Per unit cell of bonds in this setup, the energy per unit length is given by

$$
E_{\mathrm{L}}=\frac{2}{r_{0}}\left[\phi_{\text {weak }}(D)+\phi_{\text {weak }}(\tilde{D})+\ldots\right]
$$

where

$$
\phi_{\text {weak }}(D)=-\epsilon \text {. }
$$

The distance to second nearest neighbors is

$$
\tilde{D}=\frac{\Delta D}{\cos (\tilde{\phi})}
$$

where

$$
\tilde{\phi}=\tan ^{-1}\left(\frac{3 r_{0}}{2 \Delta D}\right) .
$$

Similar calculations can be done for the third, fourth, etc. nearest neighbors.

The numerical value for adhesive strength of two $(5,5)$ CNTs from MD simulation is $E_{\mathrm{L}}=2.31 \times 10^{-10} \mathrm{~J} / \mathrm{m}$.
The parameter $\epsilon$ in the mesoscopic model is chosen so that the atomistic and mesoscopic model feature the same adhesion energy per unit length. For nearest neighbors only, we find

$$
\epsilon=\frac{E_{1} r_{0}}{2}
$$

For more than one nearest neighbors in the case of larger cutoff radius,

$$
\epsilon=\frac{E_{\mathrm{L}} r_{0}}{2}\left[\left(1+\pi^{(2)}+\pi^{(3)}+\ldots\right)^{-1}\right],
$$

where $\pi=\phi_{\text {weak }}(\tilde{D}) / \phi_{\text {weak }}(D)$. We define term $(1+$ $\left.\pi^{(2)}+\pi^{(3)}+\ldots+\pi^{(N)}\right)=\beta^{(N)}$ and find that $\beta^{(6)} \approx 1.0988$, leading to $\epsilon \approx 15.1 \mathrm{kcal} / \mathrm{mol}$, with a cutoff distance at $r_{\text {cut }}=60 \AA$.

The maximum force to break a "weak" dispersive bond between two CNTs is given by

$$
F_{\max , \mathrm{LJ}}=\frac{\Delta \cdot \epsilon}{\sigma}
$$

where $\Delta \approx 2.394$ is a numerical constant. From Eq. (20), we estimate $F_{\text {max,LJ }} \approx 3.866 \mathrm{kcal} / \mathrm{mol} / \AA$, corresponding to approximately $268.62 \mathrm{pN}$. This leads to an adhesion shear strength per unit length

$$
\tau_{\max }=\frac{F_{\max , \mathrm{LJ}}}{\delta L} \approx 26.86 \mathrm{pN} / \AA
$$

\section{Tensile spring parameter}

The tensile spring constant is determined from various calculations of stretch versus deformation while being constrained to the regime of small loads and consequently small displacements.

The nonlinear elastic effects observed in MD calculations are included by defining the parameter $k_{\mathrm{T}}$ dependent on deformation state. We note that material nonlinearities may be crucial in capturing the essential fracture and deformation physics, representing the chemical effects as atomic bonds are stretched and broken. ${ }^{53,54,56}$

To develop the atomic interactions, we fit the forcestretch response to the stress-strain response obtained in full atomistic calculations [see Eq. (8b)]. The spring constant $k_{\mathrm{T}}^{(0)}$ for small deformation calculated from Young's modulus for small deformation is

$$
k_{\mathrm{T}}^{(0)}=\frac{A_{\mathrm{c}}}{L_{0}} E
$$

We find that $k_{\mathrm{T}}^{(0)} \approx 1000 \mathrm{kcal} / \mathrm{mol} / \AA^{2}$. For large deformation, the parameter $k_{\mathrm{T}}^{(1)} \approx 700 \mathrm{kcal} / \mathrm{mol} / \AA^{2}$ is determined by fitting the mesoscopic stress-strain results to the MD stress-strain curve.

The goal is to reproduce the small-strain elastic response up to approximately $5 \%$ tensile strain, as well as 
the fracture strength and fracture strain. This simple meoscopic bilinear model using $k_{\mathrm{T}}^{(0)}$ and $k_{\mathrm{T}}^{(1)}$ some of the hyperelastic effects and the fracture behavior to be captured. We note that the choice of $k_{\mathrm{T}}^{(1)}$ is largely dictated by the quantitative values of the fracture strain and fracture stress.

The model allows information about breaking of chemical bonds to be transported into the mesoscopic molecular scale model.

\section{Bending spring parameter}

Using an argument of energy conservation between the atomistic and the mesoscale model, we arrive at an expression for the bending stiffness parameter

$$
\mathrm{k}_{\mathrm{B}}=\frac{48 \cdot E I \cdot d^{2}}{8 r_{0}^{3}\left(\theta-\varphi_{0}\right)^{2}},
$$

where $\theta=2 \tan ^{-1}\left(d / r_{0}\right)$ and $d=F_{\text {appl }} 8 r_{0}^{3} /(48 \cdot E I)$. Note that $\theta$ is the angle corresponding to the displacement $d$ resulting from an applied force $F_{\text {appl }}$. We find that $\mathrm{k}_{\mathrm{B}} \approx$ $14300 \mathrm{kcal} / \mathrm{mol} / \mathrm{rad}^{2}$. These expressions are valid for small deformation.

\section{B. Summary of the mesoscopic model and its numerical implementation}

Table I summarizes all parameters used in the mesoscopic model.

The models are implemented in the massively parallelized modeling code LAMMPS ${ }^{57}$ (http://www.cs.sandia. gov/ sjplimp/lammps.html), capable of running on large computing clusters. The example calculations reported here are carried out on single CPU Linux workstations. The LAMMPS code was extended to enable treatment of the molecular interactions discussed in Sec. III. A and other loading conditions as described later.

\section{APPLICATIONS AND NUMERICAL RESULTS}

Here we report applications of the mesoscale model to describe the mechanics and self-assembly of CNTs. We

TABLE I. Summary of mesoscopic parameters derived from atomistic modeling, corresponding to Eqs. (10), (13), (14), and (15), as well as the discussion presented throughout Sec. IV.

\begin{tabular}{lr}
\hline \hline \multicolumn{1}{c}{ Parameter } & Numerical value \\
\hline Equilibrium bead distance $r_{0}($ in $\AA$ ) & 10.00 \\
Tensile stiffness parameter $k_{\mathrm{T}}^{(0)}\left(\right.$ in $\left.\mathrm{kcal} / \mathrm{mol} / \AA^{2}\right)$ & 1000.00 \\
Tensile stiffness parameter $k_{\mathrm{T}}^{(1)}\left(\right.$ in $\left.\mathrm{kcal} / \mathrm{mol} / \AA^{2}\right)$ & 700.00 \\
Hyperelastic parameter $r_{1}($ in $\AA$ ) & 10.50 \\
Fracture parameter $r_{\text {break }}$ (in $\AA$ ) & 13.20 \\
Equilibrium angle $\varphi_{0}$ (in degrees) & 180.00 \\
Bending stiffness parameter $\mathrm{k}_{\mathrm{B}}\left(\right.$ in $\left.\mathrm{kcal} / \mathrm{mol} / \AA^{2}\right)$ & 14300.00 \\
Dispersive parameter $\epsilon$ (in $\mathrm{kcal} / \mathrm{mol})$ & 15.10 \\
Dispersive parameter $\sigma$ (in $\AA$ ) & 9.35 \\
\hline \hline
\end{tabular}

focus on three cases: (i) a simple validation calculation to compare the mesoscale model to the full atomistic results; (ii) a study of self-folding of CNTs, the stability of self-folded CNTs, and self-assembly of two CNTs; and (iii) a study of bundles of CNTs subject to compressive and tensile loading, as well as the response to nanoindentation.

\section{A. Validation: Tensile test of a long CNT}

The first calculation was a validation of the mesoscale model. We implemented the loading case shown in Fig. 2(a) (tensile loading of a single CNT). The stress versus strain as obtained using the mesoscopic model is shown in Fig. 3 (dark line). The fracture strain and fracture stress agree well with the full atomistic result.

\section{B. Self-folding of CNTs and self-assembly processes}

\section{Self-assembly of a single tube: Self-folding} into racket-structures

Large-aspect ratio CNTs are extremely flexible and can be deformed into almost arbitrary shapes with relatively small energetical effort. Different CNTs attract each other via vdW forces. If different parts of the tube come sufficiently close, these attractive forces should also be present and can form self-folded structures of CNTs. Such self-folded structures of CNTs with extremely large aspect ratio were first observed in MD simulations of $(5,5)$ CNTs using a hybrid Tersoff/LJ model. ${ }^{42}$ It was suggested that different parts of highly flexible CNTs can be brought into contact either by thermal fluctuations that lead to bending of the tube or by bending resulting from application of external forces.

Here we used the mesoscopic model to investigate the stability and self-assembly properties of such racket-like self-folded CNTs. To induce self-folding, we applied an external force to the ends of the CNT, following the procedure described in Ref. 42. Once two regions of the $\mathrm{CNT}$ are in contact, the long-range vdW interactions lead to an attractive force that causes the parts of the tube to align. As a result, self-folded racket-like structures are formed. Results of mesoscale simulations are shown in Fig. 6 for two different CNT lengths. Our mesoscopic model is capable of reproducing the results of full MD simulations at a fraction of the computational cost.

At the intersection of the straight aligned part and the bent region, we observe a characteristic crossover of the lower and upper part of the tube, forming an angle close to $90^{\circ}$. This feature was also observed in full atomistic studies of the same system (see Fig. 1).

The stability of the folded structure is governed by the balance of energy required to bend the CNT and energy gained by formation of weak vdW "bonds." The bending energy is proportional to $E I$, and the adhesion energy is 
(a)

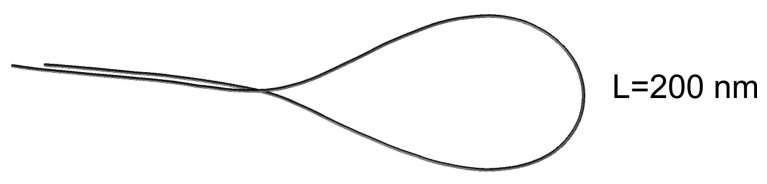

(b)

$\mathrm{L}=300 \mathrm{~nm}$

FIG. 6. Snapshots of folded CNTs, for different molecular lengths: (a) $200 \mathrm{~nm}$ and (b) $300 \mathrm{~nm}$. The self-folded structure obtained by mesoscale modeling is quite similar to the results obtained from full atomistic studies (see Fig. 1).

proportional to $\gamma$. In the following, $\gamma_{0}$ denotes the adhesion energy of two CNTs in vacuum (see the calculation presented in Sec. II. D). To assess the stability of the self-folded CNT, it is essential to understand how changes in the adhesion energy influence the structure.

The mesocale model is used to investigate the stability of the folded structure when the adhesion strength is varied. Such variations may be induced by putting the folded CNT into solution, which effectively changes the value of $\gamma$. The contact length is defined as $L_{\mathrm{c}}$, and the free length is defined as $L_{\mathrm{f}}=L-2 L_{\mathrm{c}}$ with the total length $L$ (see the schematic illustration in Fig. 7). Figure 8 shows the contact length and free length of CNT adhesion in a racket-like structure as a function of varying $\gamma$. The results are shown normalized with respect to $\gamma_{0}$, the original adhesion strength for $(5,5)$ CNTs in vacuum. The results suggest that for $\gamma_{1}=0.265 \gamma_{0}$, the critical length for stability of the racket-like CNT is $L_{\chi} \approx 300 \mathrm{~nm}$.

As a first order approximation, we assume that

$$
L_{\chi} \sim \frac{E I}{\gamma}
$$

The physical motivation is based on the fact that the critical length is given by a competition of bending energy (proportional to $E I$ ) and adhesion energy (proportional to $\gamma$ ). Note that the scaling law used in Eq. (15) can also be obtained from beam elasticity analysis. For CNTs with identical bending stiffness, the simple scaling law given by Eq. (24) can be used to estimate the critical CNT length for the original adhesion strength for different conditions. For example, for variations in length,

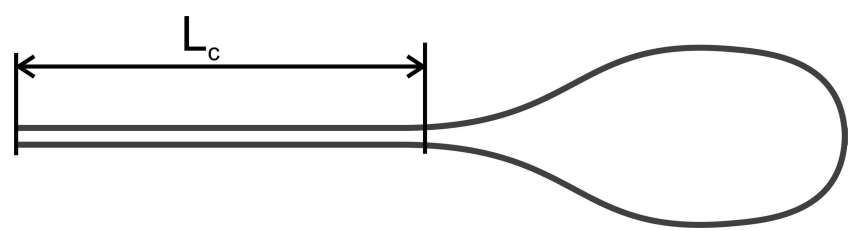

FIG. 7. Geometry of the racket-like CNT structure (schematic drawing). The contact length is defined as $L_{\mathrm{c}}$, the free length is defined as $L_{\mathrm{f}}=L-2 L_{\mathrm{c}}$ with the total CNT length $L$.

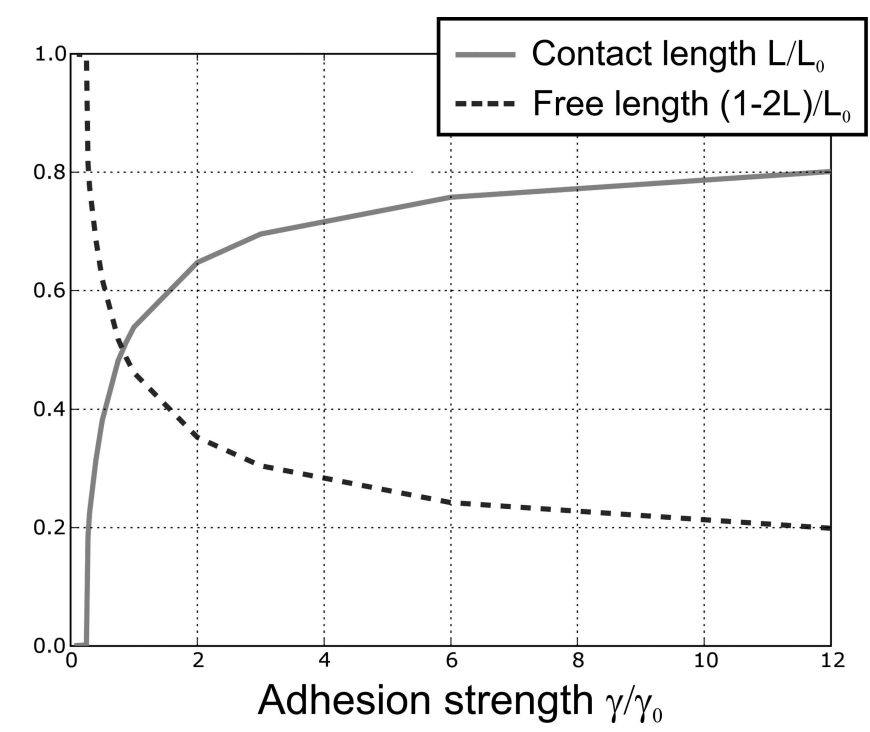

FIG. 8. Contact length and free length of a racket CNT as a function of adhesive strength. This result suggests that even if the adhesion strength is reduced (e.g.,due to solvents), the folded structure remains stable. If the adhesion strength is increased, the contact length increases, approaching half the CNT length for extremely large values. The CNT unfolds for $\gamma \approx 0.265 \gamma_{0}$.

$$
L_{\chi, 0} \sim \frac{\gamma_{1}}{\gamma_{0}} L_{\chi, 1}
$$

For the present case, we estimate $L_{\chi} \approx 78.75 \mathrm{~nm}$. Similarly, for fixed adhesion strength but variations in the bending stiffness (for example, due to changes in radius, or under consideration of multi-wall CNTs),

$$
L_{\chi, 0} \sim \frac{E I_{0}}{E I_{1}} L_{\chi, 1}
$$

The scaling laws given in Eqs. (24)-(26) provide some insight into the stability of racket-type structures for different bond lengths.

We also investigated the stability of racket-like CNT structures with respect to temperature changes. We focused on changes of the adhesion length $L_{\mathrm{c}}$ as a function of temperature. From the mesoscale simulations, we find that the average adhesion length can be expressed as a combination of a median value $L_{\mathrm{c}, \mathrm{m}}(T)$ and fluctuations in the adhesion length, denoted by $\delta L_{\mathrm{c}}(T)$

$$
L_{\mathrm{c}}=L_{\mathrm{c}, \mathrm{m}}(T) \pm \delta L_{\mathrm{c}}(T)
$$

providing a simple description of the opening-closing breathing mode found in the simulations (see Fig. 9).

From simulations with slowly increasing temperature (temperature control using a Nosé-Hoover algorithm), we observe that fluctuations of the adhesion length $\delta L_{\mathrm{c}}(T)$ increase with temperature, along with an increase in the average adhesion length $L_{\mathrm{c}, \mathrm{m}}(T)$. These results are shown in Fig. 9.

We note that the quantity $L_{\mathrm{c}, \max }=L_{\mathrm{c}, \mathrm{m}}(T)+\delta L_{\mathrm{c}}(T)$ 


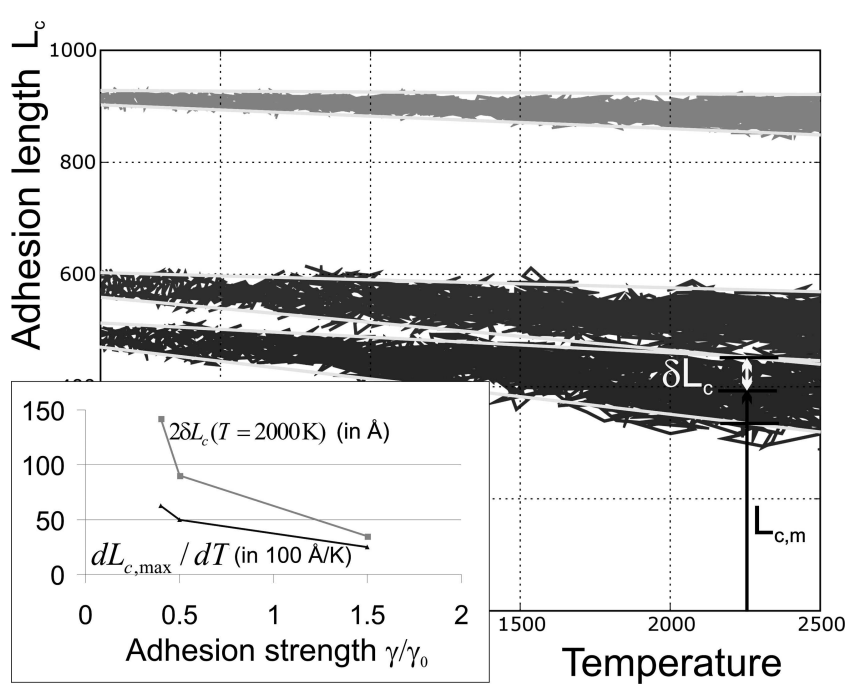

FIG. 9. Adhesion length as a function of temperature for three different values of the adhesion strength. The higher the temperature, the more fluctuations are observed in the adhesion length. The inlay plot depicts the fluctuation width as a function of adhesion strength, as well as the derivative of the adhesion length with respect to changes in temperature.

may be critical for unfolding due to thermal motion of the CNT. For example, for $\gamma=0.4 \gamma_{0}$, we find a $20 \%$ increase over a $2000 \mathrm{~K}$ temperature interval. Additional results are shown in the inlay in Fig. 9. This suggests that temperature increase could induce unfolding of the racket-like structure, only if the adhesion length is close to the critical CNT length so that the decrease in adhesion length can lead to the instability. This result further confirms earlier claims that self-folded structures can be extremely stable, with evaporation occurring before unfolding if the CNT is long enough, thus providing significant contact length. ${ }^{42}$

We also carried out calculations of CNTs in vacuum where no external forces or velocity pushes applied to investigate if the CNT would self-fold without mechanical stimulation. In this case, we did not observe selffolding of the CNT, even for lengths up to $1500 \mathrm{~nm}$, and over simulation times of $0.37 \mu \mathrm{s}$. Because this CNT length is already larger than the longest types typically found in experiments, we expect that the self-folding process requires external stimulation. This behavior can be explained by the fact that the CNT is still around one order of magnitude below its persistence length $(\xi \approx$ $1.61 \times 10^{-5} \mathrm{~m}$ versus $1500 \mathrm{~nm}$ ).

We note that such parametric and systematic studies would not have been possible with pure atomistic studies due to constraints in both length and time scale. Additional investigations will be left to future studies.

\section{Self-assembly of two CNTs}

Our mesoscale model can also be used to study the dynamic interaction of two CNTs approaching each other (a)

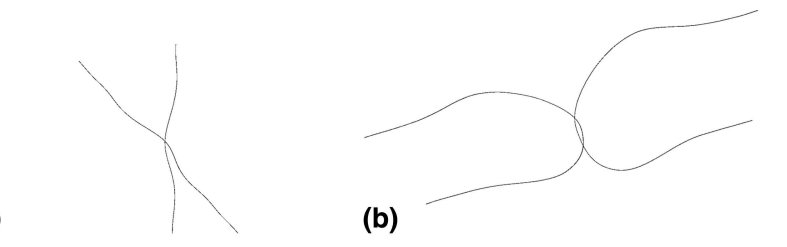

(c)
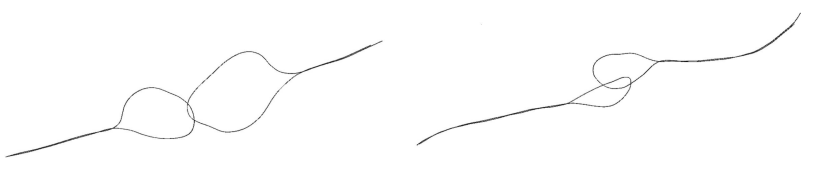

(d)

(e)

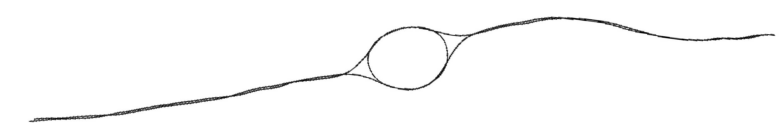

FIG. 10. Dynamical assembly of two CNTs. In this model, two carbon nanotubes oriented as indicated in (a) are moving toward each other at constant speed. Once they approach each other, interactions between the two tubes set in, leading to formation of two racket-like structures. Eventually this assembly forms a rather stable conglomerated in which the two radial heads of the racket-like structures assemble and align due to the dispersive interactions.

at a constant velocity. Figure 10 shows the simulation results of two CNTs rotated at $90^{\circ}$ with respect to each other. The two CNTs approach each other at a constant speed. We find that the two CNTs eventually assemble into a structure composed of two racket-like structures, as shown in Fig. 10(e).

Such folding and assembly mechanisms may occur in systems composed of a large number of CNTs.

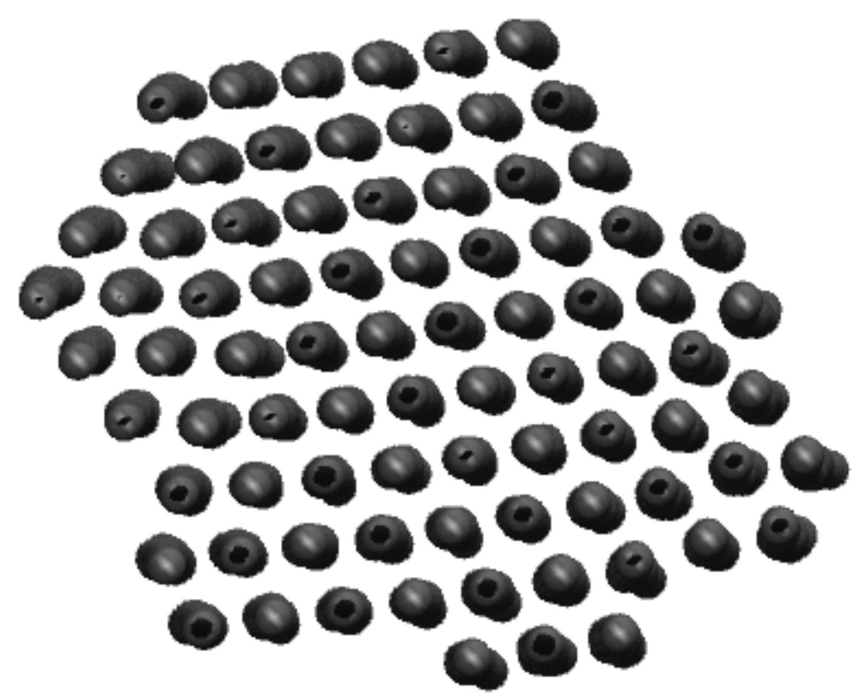

FIG. 11. Bundles of CNTs. Cross-sectional view of a bundle of CNTs forming a nanowire, with 81 CNTs. This material forms a twodimensional hexagonal structure. We note some crystal defects in this case. This also shows the cross-sectional shape of the CNT nanowire. The equilibrium shape features straight surface structures. Some crystaltype defects such as twin grain boundaries exist in the equilibrated structure. Similar structural features have been observed in allatomistic studies of bundles of CNTs. ${ }^{42}$ 


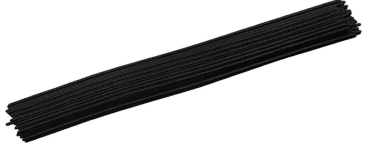

(a)

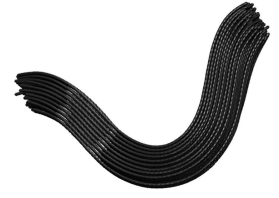

(d) (e)

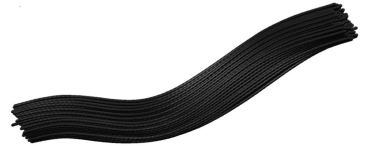

(b)

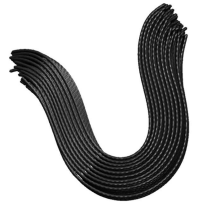

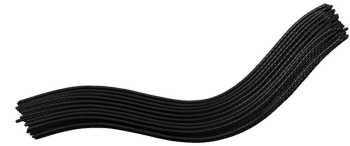

(c)

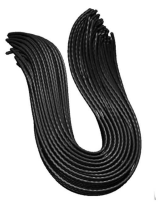

(f)

FIG. 12. Response of a CNT bundle to mechanical compressive loading (cross-sectional view of this bundle, see Fig. 10). Even for relatively small strains, the structure starts to buckle, eventually leading to significantly deformed and buckled shapes. The figure shows several snapshots as the compressive load is increased.
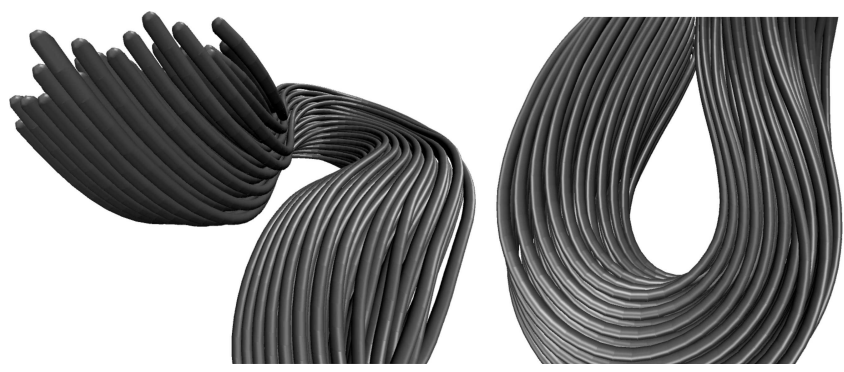

FIG. 13. Detailed view of a highly deformed CNT bundle under compressive load.

\section{Behavior of CNT bundles under mechanical stimulation}

\section{Compressive and tensile deformation of a CNT bundle}

In this section, we focus on the assembly and mechanics of bundles of CNTs. Figure 11 depicts a cross-sectional view of a CNT bundle, indicating that the tubes assemble into a triangular lattice. This agrees with earlier results obtained by full atomistic simulation. ${ }^{42}$

After equilibration of the structure, we applied compressive loading as indicated in Fig. 3(d). The results of this simulation are shown in Fig. 12. Figure 13 shows a detailed view of the deformed structure.

Similar computational experiments have also been carried out to model the deformation of CNT bundles under tensile loading (results not shown). We found that the maximum tensile strain of the CNT bundle is $29 \%$, which is close to that of a single CNT. Poisson's ratio is $v \approx$ 0.38 for small strain, reduces at larger strains, and approaches $v \approx 0.29$ close to the breaking point.

\section{Bending and fracture of a CNT bundle}

Results of deforming a CNT bundle that consists of $9(5,5)$ CNTs of $80 \mathrm{~nm}$ length each under loading of a

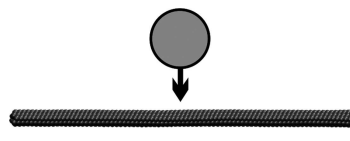

(a)

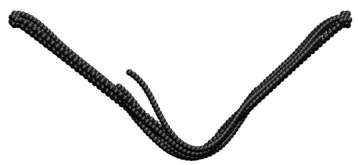

(d)

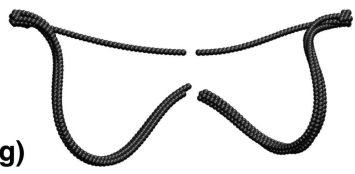

(b)
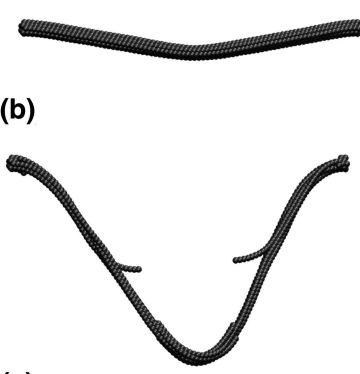

(e)

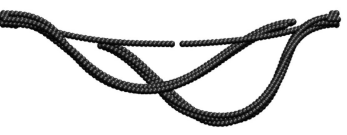

(h)

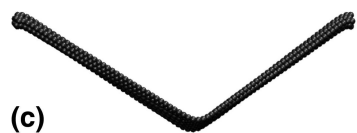

(c)

(f)

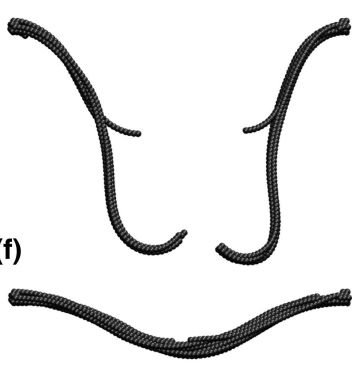

(i)

FIG. 14. Bending of a CNT bundle due to an indenter leading to fracture. The CNT bundle consists of nine $(5,5) \mathrm{CNTs}$ of $80 \mathrm{~nm}$ length each [loading case as shown in Fig. 1(c)]. (a-c) The bundle quickly goes into a bending mode, (d,e) leading to fracture of individual CNTs, (f) followed by rupture of the entire bundle. After rupture of the entire bundle and after the indenter has passed, (g-i) the structure re-assembles into a permanently (plastically) deformed shape. Note that in this case, the CNTs have fractured but are held together by dispersive interactions. 


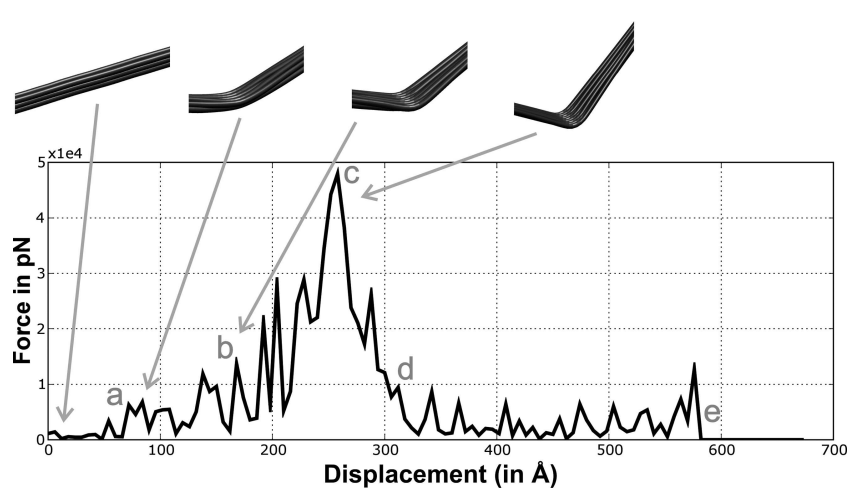

FIG. 15. Force versus displacement during indentation by a cylindrical indenter. Flattening of the cylindrically shaped bundle occurs between points $a$ and $b$, leading to increased displacement without significantly increased force. After point $b$, the bundle is completely flattened, leading to continuous force increase afterward. At point $\mathrm{c}$, the maximum load is reached, leading to rupture of individual CNTs in the bundle. As a consequence, the force decreases with increasing indenter displacement between points $\mathrm{c}$ and $\mathrm{d}$. We observe an approximately constant load between points $\mathrm{d}$ and e due to shear disentanglement of the bundle. At point e, the indenter has completely passed the bundle, leaving a zero force. The maximum indentation force is approximately $5 \times 10^{10} \mathrm{pN}$ (point c).

spherical indenter are shown in Fig. 14. The loading case is presented in Fig. 2(c).

The bundle quickly goes into a bending mode, leading to fracture of individual CNTs [Fig. 14(e)], followed by rupture of the entire bundle [Fig. 14(f)]. After rupture of the entire bundle and after the indenter has passed, the structure re-assembles into a permanently (plastically) deformed shape [Fig. 14(i)]. Note that in this case, all CNTs in the bundle have been fractured but are held together by dispersive interactions.

Figure 15 shows a load-displacement curve for this deformation case. After the indenter approaches the bundle, we observe significant flattening of the cylindrically shaped bundle (between points a and b). In this regime, we find increased displacement without significantly increased force. After point $b$, the bundle is completely flattened, leading to continuous force increase when the indenter moves further into the bundle. At point $\mathrm{c}$, the maximum load is reached, leading to rupture of individual CNTs in the bundle. As a consequence, the force decreases with increasing indenter displacement between points $\mathrm{c}$ and $\mathrm{d}$. We observe an approximately constant load between points $\mathrm{d}$ and e due to shear disentanglement of the bundle. At point $\mathrm{e}$, the indenter has completely passed the bundle, leaving a zero net force. The maximum indentation force is approximately $5 \times 10^{4} \mathrm{pN}$.

These studies allow detailed understanding of the molecular mechanisms leading to failure of bundles and assemblies of CNTs. Clearly, atomistic models of these cases are computationally much more expensive and limited in terms of the accessible time scales. Additional investigations are left for future work.

\section{DISCUSSION, CONCLUSION AND OUTLOOK}

In this paper, we have reported atomistic modeling to calculate the elastic, plastic, and fracture properties of individual CNTs, using a hierarchy of atomistic and mesoscale modeling approaches. In this section, we summarize the main contributions of this paper.

We developed a simple mesoscopic model to describe the mechanical properties and self-assembly mechanisms of CNTs with ultra large aspect ratio. The parameters of the mesoscopic model were derived completely from the results of full atomistic modeling.

Our mesoscopic model is capable of reproducing the full atomistic results of single tube tensile load cases (see comparison shown in Fig. 3). The mesoscopic model can reach time and length scales not accessible to the full atomistic model but still includes information about the fracture mechanics of individual CNTs.

We reported an analysis of the stability of self-folded racket-like CNTs (see Fig. 6), in particular, focusing on the stability due to variations of the adhesion strength. We found that even when the adhesion strength is reduced, for example due to solvents, the self-folded structure is remarkably stable. Additional investigations shed light on structural changes due to increased temperature (Figs. 8 and 9). By systematically varying the adhesion strength, it is possible to control the dynamic equilibrium radius or adhesion length of the self-folded CNT. Such variations could be induced by choosing different solvents. This could lead to an application of the self-folded CNT as a sensor for different chemical environments.

We showed that two CNTs moving toward each other may assemble into a racket-like shape, forming conglomerated structures (see Fig. 10).

Studies of bundles of CNTs enable investigations of the response to compressive, tensile, and bending loading. We find that bundles of CNTs start to buckle once the compressive strain reaches a critical value. The fracture properties govern the response of a CNT bundle under bending deformation, induced by an indenter. We could link microscopic events with the overall shape of the force-displacement curve (see Figs. 14 and 15).

We note that the mesoscopic model can be straightforwardly implemented for CNTs other than those studied here, including multiwalled CNTs.

We believe that reactive modeling that takes into account the complexity of chemical bonding may be critical to understanding the fracture and deformation behavior of other nanoscale materials. Indeed, our results suggest that it is critical to include the correct description of the bond behavior and breaking processes at large bond stretch, information stemming from the quantum 
chemical details of bonding. In our current model, this information is included in the Tersoff force field parameters, ${ }^{45}$ which in turn influence the parameter of the mesoscopic model within a hierarchical multiscale scheme. Without correct description of fracture processes, the large-deformation regime of CNTs that induces fracture - as shown in studies of nanoindentation of CNT bundles-cannot be described correctly.

We note that new reactive force fields represent an alternative to Tersoff-type potentials as used in this study. These force fields provide a more accurate description of the bond breaking and formation processes in predominantly covalently bonded materials. ${ }^{58-60} \mathrm{We}$ emphasize that our method can immediately be applied to include atomistic results based on more accurate potentials or even first-principles-based calculations. We leave such studies to future work.

The agreement between the atomistic and mesoscale models could be improved by using a more elaborate Ansatz for the stretching potential than the bilinear technique, possibly using spline functions. This could improve the agreement between the mesoscale model and the atomistic model in particular at strain levels between $15 \%$ and $30 \%$ tensile strain. This may be particularly useful when more accurate atomistic data, for example obtained using reactive force fields, are used for the training of the mesoscale parameters.

Compared to the full atomistic model, the mesoscopic description is not capable to reproduce certain atomisticscale aspects of fracture and deformation. This includes formation of 5-7 Stone-Wales-type defects or chemical reactions that may occur during the deformation processes.

The simple mesoscale model reported here opens up several possibilities for future studies, particularly at the scale of CNT bundles, as well as applications that focus on integrated composite structures. Results from mesoscopic modeling could be used in the development of new constitutive equations that can be used in continuum or finite element studies. For example, the mesoscopic model can be used to describe tensile experiments of pulling and deformation of CNTs with extremely large aspect ratios, naturally including the effects of entropy on their elastic behavior.

We have demonstrated that it is unlikely that the selffolding of CNTs into racket-structures will occur only due to entropic fluctuations, because the most experimentally synthesized CNTs are well below the persistence length. Thus, thermal fluctuations are not sufficiently large to induce contact of different areas of a single tube. Our modeling clearly shows that such contact is a critical condition to induce self-folding. We believe that two length scales exist that characterize the entropic properties of ultralong tubes. First, the persistence length $\xi$ describes the amount of thermal fluctuations into bending modes, at a given temperature. Secondly, there exists a critical length $L_{x}$ of CNTs to allow stable self-folded structures. This length scale is proportional to the bending stiffness and inversely proportional to the adhesion energy, as discussed in Sec. IV. B. 1. We find that typically $L_{\chi}<\xi$, suggesting that self-folding is possible at CNT lengths much below the persistence length.

It remains an open question as to what the effects of the folded structure are on optical or electronic properties. The head-radius is typically on the order of a few tens of nanometers; thus interesting optical properties may be induced by these new materials. Other selfassembly processes - as demonstrated in Fig. 10-may lead to interesting new materials and novel nanostructures. Techniques to functionalize the CNT surface and induce covalent cross-links, preferred adhesion domains, or other methods could help building complex nano-scale structures and materials.

\section{ACKNOWLEDGMENTS}

M.J.B. acknowledges generous support from MIT's CEE Department. The calculations and the analysis were carried out using a Linux computer at MIT's Atomistic Mechanics Modeling Laboratory. M.J.B. acknowledges helpful discussions with V. Molinero at the California Institute of Technology, $\mathrm{H}$. Gao at Brown University, and Y. Huang at UIUC. Visualization was carried out using the VMD visualization package. ${ }^{61}$

\section{REFERENCES}

1. S. Iijima: Helical microtubules of graphitic carbon. Nature $\mathbf{3 5 4}, 56$ (1991).

2. S.E. Moulton, A.I. Minett, and G.G. Wallace: Carbon nanotube based electronic and electrochemical sensors. Sens. Lett. 3, 183 (2005).

3. A. Modi, N. Koratkar, E. Lass, B.Q. Wei, and P.M. Ajayan: Miniaturized gas ionization sensors using carbon nanotubes. $\underline{\mathrm{Na}}$ ture 424, 171 (2003).

4. V. Sazonova, Y. Yaish, H. Ustunel, D. Roundy, T.A. Arias, and P.L. McEuen: A tunable carbon nanotube electromechanical oscillator. Nature 431, 284 (2004).

5. H. Jiang, M.F. Yu, B. Liu, and Y. Huang: Intrinsic energy loss mechanisms in a cantilevered carbon nanotube beam oscillator. Phvs. Rev. Lett. 93, 185501 (2004).

6. J.Y. Huang, S. Chen, Z.Q. Wang, K. Kempa, Y.M. Wang, S.H. Jo, G. Chen, M.S. Dresselhaus, and Z.F. Ren: Superplastic carbon nanotubes - Conditions have been discovered that allow extensive deformation of rigid single-walled nanotubes. Nature 439, 281 $\underline{(2006)}$.

7. W.D. Zhang, F. Yang, and P.Y. Gu: Carbon nanotubes grow to pillars. Nanotechnology 16, 2442 (2005).

8. H. Gao, Y. Kong, D. Cui, and C.S. Ozkan: Spontaneous insertion of DNA oligonucleotides into carbon nanotubes. Nano Lett. 3, 471 $(2003)$.

9. X. Guo, J.B. Wang, and H.W. Zhang: Mechanical properties of single-walled carbon nanotubes based on higher order CauchyBorn rule. Int. J. Solids Struct. 43, 1276 (2006). 
10. H. Lu and L. Zhang: Analysis of localized failure of single-wall carbon nanotubes. Comput. Mater. Sci. 35, 432 (2006).

11. D.L. Shi, X.Q. Feng, H.Q. Jiang, Y.Y. Huang, and K.C. Hwang: Multiscale analysis of fracture of carbon nanotubes embedded in composites. Int. J. Fract. 134, 369 (2005).

12. C.Y. Li, R.S. Ruoff, and T.W. Chou: Modeling of carbon nanotube clamping in tensile tests. Compos. Sci. Technol. 65, 2407 (2005).

13. T. Natsuki and M. Endo: Stress simulation of carbon nanotubes in tension and compression. Carbon 42, 2147 (2004).

14. L.C. Qin, X.L. Zhao, K. Hirahara, Y. Miyamoto, Y. Ando, and S. Iijima: Materials science-The smallest carbon nanotube. $\mathrm{Na}$ ture 408, 50 (2000).

15. P.M. Ajayan and S. Iijima: Smallest carbon nanotube. Nature 358. 23 (1992).

16. B.I. Yakobson, C.J. Brabec, and J. Bernholc: Nanomechanics of carbon tubes: Instabilities beyond linear response. Phvs. Rev. Lett. 76, 2511 (1996).

17. T. Ozaki, Y. Iwasa, and T. Mitani: Stiffness of single-walled carbon nanotubes under large strain. Phys. Rev. Lett. 84, 1712 $\underline{(2000)}$.

18. G. Dereli and C. Ozdogan: Structural stability and energetics of single-walled carbon nanotubes under uniaxial strain. Phvs. Rev. B 67, 035416 (2003).

19. C.Q. Ru: Axially compressed buckling of a doublewalled carbon nanotube embedded in an elastic medium. J. Mech. Phys. Solids 49, 1265 (2001).

20. B. Ni, S.B. Sinnott, P.T. Mikulski, and J.A. Harrison: Compression of carbon nanotubes filled with $\mathrm{C}-60, \mathrm{CH} 4$, or Ne: Predictions from molecular dynamics simulations. Phys. Rev. Lett. 88, 205505 (2002).

21. O. Hod, E. Rabani, and R. Baer: Carbon nanotube closed-ring structures. Phys. Rev. B 67, 195408 (2003).

22. T. Hertel, R.E. Walkup, and P. Avouris: Deformation of carbon nanotubes by surface van der Waals forces. Phvs. Rev. B $\mathbf{5 8}$ 13870 (1998).

23. H. Ulbricht, G. Moos, and T. Hertel: Interaction of C-60 with carbon nanotubes and graphite. Phvs. Rev. Lett. 90, 095501 $\underline{(2003)}$.

24. M. Arroyo and T. Belytschko: Continuum-mechanics modeling and simulation of carbon nanotubes. Meccanica 40, 455 (2005).

25. H. Jiang, Y. Huang, and K.C. Hwang: A finite-temperature continuum theory based on interatomic potentials. J. Eng. Mater. Technol. Trans. ASME 127, 408 (2005).

26. P. Zhang, Y. Huang, H. Gao, and K.C. Hwang: Fracture nucleation in single-wall carbon nanotubes under tension: A continuum analysis incorporating interatomic potentials. J. Appl. Mech.Trans. ASME 69, 454 (2002).

27. S.H. Yeak, T.Y. Ng, and K.M. Liew: Multiscale modeling of carbon nanotubes under axial tension and compression. Phvs. Rev. B 72, 165401 (2005).

28. Q. Lu and B. Bhattacharya: Effect of randomly occurring StoneWales defects on mechanical properties of carbon nanotubes using atomistic simulation. Nanotechnol. 16, 555 (2005).

29. N.M. Pugno and R.S. Ruoff: Quantized fracture mechanics. $\underline{P h i-}$ los. Mag. 84, 2829 (2004).

30. M.A.L. Marques, H.E. Troiani, M. Miki-Yoshida, M. Jose-Yacaman, and A. Rubio: On the breaking of carbon nanotubes under tension. Nano Lett. 4, 811 (2004).

31. L.G. Zhou and S.Q. Shi: Molecular dynamic simulations on tensile mechanical properties of single-walled carbon nanotubes with and without hydrogen storage. Comput. Mater. Sci. 23, 166 (2002).
32. V. Molinero and W.A. Goddard: Microscopic mechanism of water diffusion in glucose glasses. Phvs. Rev. Lett. 95, 045701 (2005).

33. M.H. Lamm, T. Chen, and S.C. Glotzer: Simulated assembly of nanostructured organic/inorganic networks. Nano Lett. 3, 989 $\underline{(2003)}$.

34. P.T. Underhill and P.S. Doyle: On the coarse-graining of polymers into bead-spring chains. J. Non-Newtonian Fluid Mech. 122. 3 (2004).

35. A. Maiti, J. Wescott, and P. Kung: Nanotube-polymer composites: Insights from Flory-Huggins theory and mesoscale simulations. Mol. Simul. 31, 143 (2005).

36. A.H. Barber, S.R. Cohen, A. Eitan, L.S. Schadler, and H.D. Wagner: Fracture transitions at a carbon-nanotube/polymer interface. $A d v$. Mater. 18, 83 (2006).

37. J.V. Barth, G. Costantini, and K. Kern: Engineering atomic and molecular nanostructures at surfaces. Nature 437, 671 (2005).

38. M. Zhang, S.L. Fang, A.A. Zakhidov, S.B. Lee, A.E. Aliev, C.D. Williams, K.R. Atkinson, and R.H. Baughman: Strong, transparent, multifunctional, carbon nanotube sheets. Science $\mathbf{3 0 9}$. 1215 (2005).

39. Y. Huang, C.Y. Chiang, S.K. Lee, Y. Gao, E.L. Hu, J. De Yoreo, and A.M. Belcher: Programmable assembly of nanoarchitectures using genetically engineered viruses. Nano Lett. 5, 1429 (2005).

40. M. Hazani, F. Hennrich, M. Kappes, R. Naaman, D. Peled, V. Sidorov, and D. Shvarts: DNA-mediated self-assembly of carbon nanotube-based electronic devices. Chem. Phvs. Lett. 391. 389 (2004).

41. Q. Gu, C.D. Cheng, R. Gonela, S. Suryanarayanan, S. Anabathula, K. Dai, and D.T. Haynie: DNA nanowire fabrication. Nanotechnology 17, R14 (2006).

42. M.J. Buehler, Y. Kong, and H.J. Gao: Self-folding and unfolding of carbon nanotubes. J. Eng. Mater. Technol. 128, 3 (2006).

43. M.J. Buehler, Y. Kong, and H.J. Gao: Deformation mechanisms of very long single-wall carbon nanotubes subject to compressive loading. J. Eng. Mater. Technol. 126, 245 (2004).

44. M.P. Allen and D.J. Tildesley: Computer Simulation of Liquids (Oxford University Press, New York, 1989).

45. J. Tersoff: Empirical interatomic potentials for carbon, with applications to amorphous carbon. Phvs. Rev. Lett. 61, 2879 (1988).

46. F. Stillinger and T.A. Weber: Computer-simulation of local order in condensed phases of silicon. Phys. Rev. B 31, 5262 (1985).

47. J. Stadler, R. Mikulla, and H-R. Trebin: IMD: A software package for molecular dynamics studies on parallel computers. Int. J. Mod. Phvs. C. 8, 1131 (1997).

48. J. Roth, F. Gahler, and H-R. Trebin: A molecular dynamics run with 5.180.116.000 particles. Int. J. Mod. Phys. C. 11, 317 (2000).

49. D.H. Tsai: Virial theorem and stress calculation in moleculardynamics. J. Chem. Phvs. 70, 1375 (1979).

50. H.T. Yang, J.W. Chen, L.F. Yang, and J.M. Dong: Oscillations of local density of states in defective carbon nanotubes. Phys. Rev. B 71, 085402 (2005).

51. F. Ding: Theoretical study of the stability of defects in singlewalled carbon nanotubes as a function of their distance from the nanotube end. Phvs. Rev. B 72, 245409 (2005).

52. M.J. Buehler: Atomistic and continuum modeling of mechanical properties of collagen: Elasticity, fracture and self-assembly. J. Mater. Res. 21(8), 1947 (2006).

53. M.J. Buehler and H. Gao: Dynamical fracture instabilities due to local hyperelasticity at crack tips. Nature 439, 307 (2006).

54. M.J. Buehler, F.F. Abraham, and H. Gao: Hyperelasticity governs dynamic fracture at a critical length scale. Nature 426, 141 (2003).

55. S.L. Mayo, B.D. Olafson, and W.A. Goddard: Dreiding-A generic force-field for molecular simulations. J. Phys. Chem. 94. 8897 (1990). 
56. H. Gao: A theory of local limiting speed in dynamic fracture. J. Mech. Phvs. Solids. 44, 1453 (1996).

57. S. Plimpton: Fast parallel algorithms for short-range moleculardynamics. J. Comput. Phvs. 117, 1 (1995).

58. M.J. Buehler, A.C.T.v. Duin, and W.A. Goddard: Multi-paradigm modeling of dynamical crack propagation in silicon using the ReaxFF reactive force field. Phys. Rev. Lett. 96, 095505 (2006).

59. A.C.T.v. Duin, S. Dasgupta, F. Lorant, and W.A. Goddard:
ReaxFF: A reactive force field for hydrocarbons. J. Phys. Chem. A. 105, 9396 (2001).

60. K.D. Nielson, A.C.T.v. Duin, J. Oxgaard, W. Deng, and W.A. Goddard: Development of the ReaxFF reactive force field for describing transition metal catalyzed reactions, with application to the initial stages of the catalytic formation of carbon nanotubes. J. Phvs. Chem. A 109, 49 (2005).

61. W. Humphrey, A. Dalke, and K. Schulten: VMD: Visual molecular dynamics. J. Mol. Graphics 14, 33 (1996). 